\title{
Performance Analysis of Wavelet Transforms for Learning based Single Frame Image Super-resolution
}

\author{
Anil A. Patil \\ Department of Electronics \& Telecommunication \\ COE, Malegaon, Pune, India
}

\author{
Jyoti Singhai \\ Department of Electronics \& Communication \\ MANIT, Bhopal, India
}

\begin{abstract}
Image super resolution concept has been introduced for image enhancement in various applications. Image enhancement is crucial operation essential for reducing different possible degradations of the captured image. More sophisticated techniques are already proposed. Wavelet transform based algorithms are widely used in many applications. Wavelet transform/s is used to extrapolate missing high frequency components which improve the efficiency of an algorithm. In this paper for super resolving the images, wavelet coefficients of the unknown high resolution image are learnt from a set of high resolution training images in wavelet domain. The performance of different discrete orthogonal and a biorthogonal wavelets have been evaluated on different class of images in terms of MSE and PSNR. The outcome of this work suggests that use of $\mathrm{db} 4$ wavelet transform is appropriate for super resolution technique. The PSNR obtained with this transform outfits for other wavelet transforms.
\end{abstract}

\section{General Terms}

Image Processing

\section{Keywords}

Super-resolution(SR), Wavelet Transform, Learning Method

\section{INTRODUCTION}

In this modern era imaging techniques are being replaced by digital image processing techniques with high rate. In almost all the imaging applications high resolution (HR) images are required for analysis and diagnosis purpose and it gives fruitful results. The captured images by camera system are of aliased, blurred and noisy, which are of degraded quality. Such images are acknowledged as low resolution images (LR). The spatial resolution of the low resolution image can be improved by increasing the pixel-density or by growing the chip-size. Both the techniques enforce definite limitations. Resolution enhancement from a single observation using image interpolation technique has restricted applications since aliasing is present in the low-resolution image. The resolution of a digital imaging device is mainly limited by the number of pixels on the sensor and the optical system. Spatial resolution of the low resolution image can be improved further than the limits of physical sensor and degradations can be removed which occur during image acquisition process [1]. Tsai and Hung were first to propose Super-resolution idea in 1984. Super-resolution (SR) is simply a technique where one or more frames are used to overcome the inherent resolution limitations of currently used camera system. In this technique the low resolution image is upsampled through which spatial frequency is improved and it removes the degradations which take place during image capture. The SR can be classified in two categories as reconstruction based and learning based. In reconstruction based methods the spatial resolution is increased by proper fusion of a series of accurately registered aliased images (frames). Very high accuracy is required in the registration to be able to reconstruct the high resolution image correctly. The quality of reconstructed SR image obtained from a set of LR images depends upon the registration accuracy of the LR images [2,3]. Reconstruction based SR uses several low resolution observations to estimate motion or blur or zoom to reconstruct high resolution image [4]. Several times, single low resolution frame is available and be superresolved. Learning based methods are more suitable for the said problem. In single frame image super-resolution, a set of one or more training images of same or different types are used where nonredundant information is extrapolated from the high resolution training data set to learn the unknown high resolution image [5]. Super-resolution is a process of estimating a high resolution image from one or more lowresolution observations where handling of data at multiple resolutions is a problem, and since the wavelets are best suitable for a multiresolution analysis.

The paper is organized as follows. In section two wavelet based learning is discussed. Experimentation and result is discussed in section three. Paper concludes with section four.

\section{WAVELET BASED LEARNING}

Wavelets are used to represent data at multiple resolution level. It has been observed that time-frequency representation of signals can easily be represented by using wavelet transform. Discrete wavelet transform uses filters of different cutoff frequencies to analyze the sequence at different scales. A 2D wavelet decomposition is performed on rows by applying lowpass and highpass filter. Same operation is performed on columns. This decomposition outputs four subbands as LL, LH, HL, HH which is illustrated in Fig. 1. Here $x[n]$ is input image and $h[n]$ and $g[n]$ are low and high pass filters $[9,10]$. It decomposes digital images into some frequency sub-images which are being represented by proportional frequency resolution. This technique decomposes image into approximate and vertical details, horizontal details, and diagonal details. Thus image is decomposed into multiple levels and the high frequency components are separated out by using multilevel wavelet. The extrapolated high frequency components are used to reconstruct the high resolution image using learning techniques. Here wavelets are used for learning the best edges from a high resolution training data using the low resolution edge primitive and it is used as a prior to obtain the super resolved image. For learning, the unknown high resolution test image is decomposed at 2 levels and all the training images in the data set are decomposed at three levels [8]. The coefficients of test image and training images are compared at coarser two levels in wavelet domain. The best match finer coefficients are copied from the training image to the corresponding location at finer scale of test image. Here 
an upsampling factor is considered as 2. Fig. 2 illustrates the learning of wavelet coefficients at finer scale. In learning process the zero tree concept has been applied to get the relationship at the next coarser scale of similar orientation [7]. Each coefficient in the subband $I-I I I$ and the corresponding $2 \times 2$ blocks in the subband $I V-V I$, the $4 x 4$ block of coefficients corresponding to best match from the subband $V I I-I X$ are copied at the corresponding location of the test image. The matching of the coefficient is done on the basis of minimum absolute difference. This decomposition is used to extrapolate the missing high frequency coefficients of the test image. Thus extrapolated high frequency components are used to reconstruct the high resolution image using learning techniques.

The minimum absolute difference is obtained by using following equation:

$$
\begin{aligned}
& \hat{\imath}(a, b)=\arg \min _{l}\left[d_{I}(a, b+L / 4)-d_{I(l)}(a, b+L / 4) \mid\right. \\
& +\left|d_{I I}(a+L / 4, b)-d_{I I(l)}(a+L / 4, b)\right| \\
& +\left|d_{I I I}(a+L . / 4, b+L / 4)-d_{I I I}(l)(a+L .4, b+L .4)\right| \\
& +\sum_{p=a}^{p=a+1 q=b+1}\left|\sum_{I=b}^{b}(p, q+L / 2)-d_{I V}(l)(p, q+L / 2)\right| \\
& +\underset{\sum}{p=a} \underset{\sum=b}{p+1 q=b+1}\left|d_{V}(p+L / 2, q)-d_{V(l)}(p+L / 2, q)\right| \\
& \left.+\sum_{p=a}^{p=a+1 q=b+1} \sum_{q=b}\left|d_{V I}(p+L / 2, q+L / 2)-d_{V I}(l)(p+L / 2, q+L / 2)\right|\right]
\end{aligned}
$$

Where $l=1,2, . ., N$ and $d_{J}(l)$ denotes the wavelet coefficients for the $l^{\text {th }}$ training image at $J^{\text {th }}$ subband. For each $(a, b)$ in subbands $I-I I I$ of low resolution observation, a $4 x 4$ block of wavelet coefficients are then copied into subbands $V I I, V I I I, I X$ of the test image from subbands $V I I-I X$ of the training images where $\hat{l}(a, b)$ gives the minimum. This minimum absolute difference gives best match and helps in matching the edge primitives at low resolutions. The same procedure is repeated for each coefficient in subbands $I, I I, I I I$ of the low resolution image. In effect for each coefficient in $I-I I I, 16$ coefficients in subbands $V I I-I X$ are learned from the training set. Each $4 \times 4$ region has been learnt from different training images. When the difference $\hat{l}(a, b)$ is large, it shows that the $4 \times 4$ block does not have a good match. This specifies that edge primitive does not have its corresponding high resolution representation in the database. To avoid such false learning wavelet coefficients are customized to have the difference $\hat{l}(a, b)$ less than certain threshold.
The methodology used in this algorithm is given in steps below:

1) Decompose test image in two levels and decompose all training images in three levels in wavelet domain.

2) Take the wavelet coefficients at each location of LH, HL and HH subbands at first level and the corresponding $2 \times 2$ blocks of the second level of the test image as well as the high resolution training set.

3) Obtain the absolute difference between the wavelet coefficients in the low resolution image and the corresponding coefficients for each of the training images.

4) If absolute difference, $\hat{l}(a, b)<$ threshold, obtain the unknown high resolution wavelet coefficients (4 $\mathrm{x} 4$ block) from a training image from the corresponding subbands, else set them all zeros.

5) Repeat steps (2-4) for every detail wavelet coefficients, at first level subbands.

6) The inverse wavelet transform reconstructs the super resolved image.

In post processing histogram specification technique is used for image enhancement.

\section{EXPERIMENTATION AND RESULT}

In this work still image of particular class of size $\mathrm{M} \times \mathrm{M}$ is selected as test image. A set of high resolution training images of same class is downloaded from internet each of size $2 \mathrm{M} \times 2 \mathrm{M}$ to form a high resolution dataset, which is assessable. Such a set of downloaded high resolution images of same class is used as a training set. In this training set, 50 high resolution images are considered for the experimentation. In this experimentation four different discrete orthogonal and a biorthogonal wavelets are tested for their performance on particular class of test image. All the wavelets selected here are optimal in their resprctive family. The performance of different wavelet is evaluated on the basis of MSE and PSNR. The low resolution test image is obtained from its high resolution version through a low resolution image formation model which downsamples it by a factor of 2 [8]. This decimation model simulates the integration of light intensity that falls on the high resolution detector. Such obtained low resolution test image of size $\mathrm{M} \times \mathrm{M}$ is used for the experimentation. Figure 3(a) shows low resolution building image of size $64 \times 64$ pixels. Figure 3(b) shows the original high resolution image of size $128 \times 128$ which has prominent edges. Figure 3(c) shows same image upsampled by a factor of 2 using haar wavelet. Haar is a basic two point wavelet which is discontinuous, gives blurred edges and prominent artifacts at the edges in reconstructed image. Reconstructed image using Daubechies-4 is shown in figure 3(d). Daubechies wavelet, db4, which analysis is orthogonal, includes basic haar wavelet with the vanishing moments of number $\mathrm{N}$ and is not symmetrical. These wavelets have the ability to provide more number of vanishing moments for a given support width. The fourth order Daubechies wavelet has a vanishing moment of 4 . It decomposes the polynomial of order 3 and wavelet function analyses the signal with trends of a third order polynomial. The reconstructed image shows little blurred edges and few artifacts at the diagonal and horizontal edges. Reconstructed image shown in figure 3(e) is using Coilflets3 wavelets which is having highest number of vanishing moments, and is more symmetrical, gives compatible results with Daubechies wavelet. Very fewer 
artifacts have been observed at the diagonal edges with blur. The reconstructed image shown in figure 3(f) is using Biorthogonal spline wavelet where exact reconstruction is possible with FIR filters. Here two wavelets have been used each for decomposition and reconstruction. Noticeable artifacts have been observed with more blurred image. Reconstructed image shown in figure $3(\mathrm{~g})$ is using Discrete Meyer wavelet which is not compactly supported, gives compatible results with Biorthogonal spline wavelets with less blurred image.

Figure 4(a) shows the low resolution image of face and figure 4(b) is its original high resolution version of size $128 \times 128$ with fine details. Figure 4(c) is reconstructed image using haar wavelets where mouth, noise and eyes are blurred and artifacts have been observed at curved edges. The details of eye and teeth have been lost. Reconstructed image shown in figure 4(d) is using db4 wavelets where more details of eye and teeth can be seen with less blur, but small artifacts are present at the face edges. Reconstructed image shown in figure 4(e) is using Coilflet wavelets where fewer artifacts with some details of face have been observed. Reconstructed image shown in figure 4(f) is using Biorthogonal spline wavelets which gives fewer details and added blur have been observed in the reconstructed image. Reconstructed image shown in figure 4(g) is using Discrete Meyer wavelet which is not having the compact support gives compatible results with Biorthogonal wavelets with less blur. Table 1 illustrates the results of different wavelets for both building and face class of images. The MSE and respective PSNR in terms of $\mathrm{db}$ are shown in the table. The learning of the low resolution test image depends on the training images in the dataset. All the wavelets used to evaluate the performance have almost same computational complexity in both classes. The mean squared error and PSNR is calculated as

$$
M S E=\frac{\sum_{a, b}(S(a, b)-\hat{S}(a, b))^{2}}{\sum_{a, b}(S(a, b))^{2}}
$$

$$
P S N R=10 \log \left(\frac{255^{\wedge} 2}{M S E}\right)
$$

Where $S(a, b)$ original high resolution image and $\hat{S}(a, b)$ is reconstructed super resolved image. Figure 5(a) and 5(b) are the graphical illustration of MSE vs. Wavelets and PSNR vs. Wavelets used to assess the performance, in sequence as listed in table 1 . Daubechies-4 bare minimum MSE and utmost PSNR for building and face class of images.

\section{CONCLUSION}

The experimentation shows Daubechies-4 wavelet gives superior performance with minimum blur and least amount of artifacts at the edges for both classes of images. Asymmetry of Daubechies has pronounced in both building and face class of images. The compatible results have been observed for Coilflet3 wavelet. The result improves as the vanishing movement increases within support. To avoid artifacts at the diagonal edges, directional filters can be used to improve the efficiency of the algorithm. The learning of the low resolution image depends upon how much training dataset is strong.

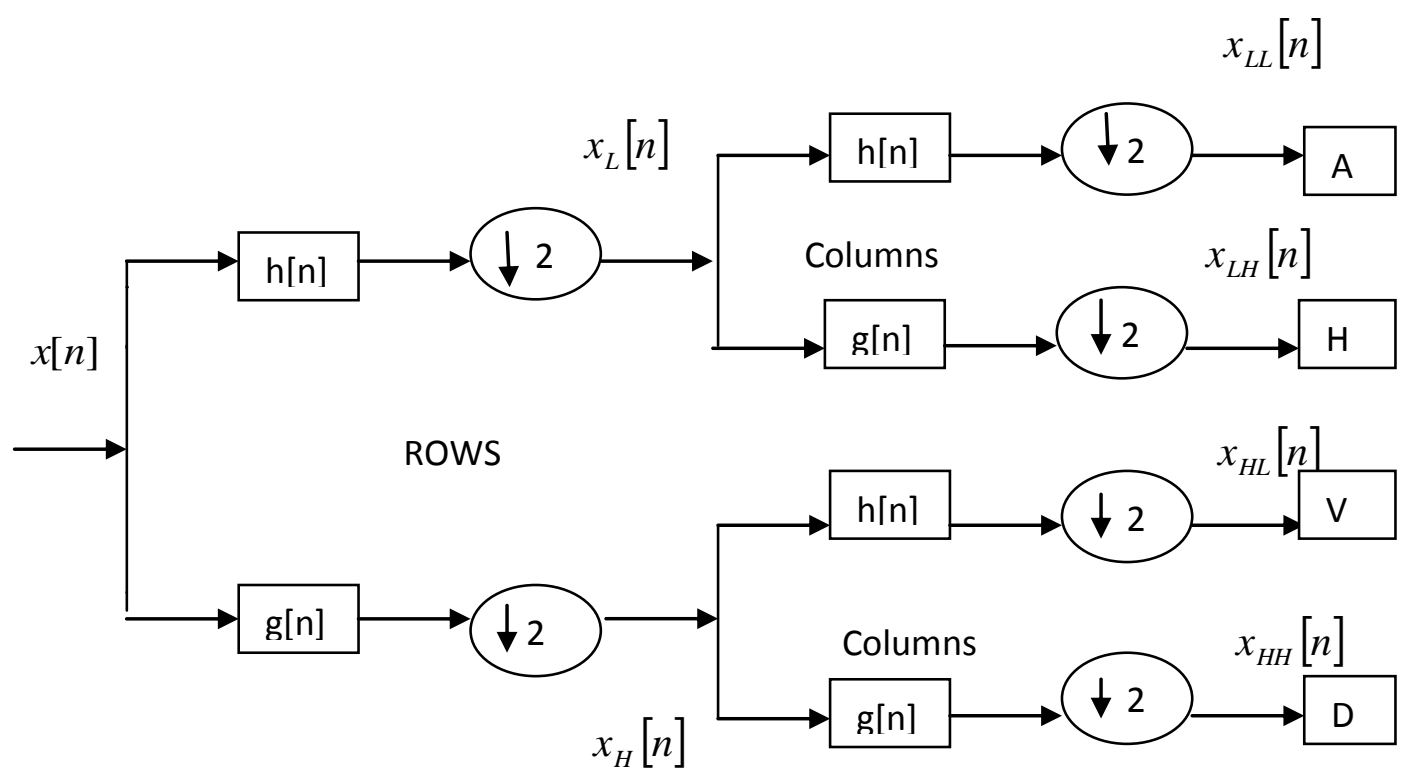

Fig 1: Illustration of wavelet subband decomposition 


\begin{tabular}{|c|c|c|c|}
\hline 0 & 1 & \multirow[t]{2}{*}{ IV } & \multirow{3}{*}{ VII } \\
\hline II & III & & \\
\hline V & & VI & \\
\hline \multicolumn{3}{|c|}{ VIII } & IX \\
\hline
\end{tabular}

Test image

\begin{tabular}{|c|c|c|c|}
\hline O I & IV & \multirow{2}{*}{ VII } \\
\hline II & III & \\
\hline V & VI & \\
\hline & & \\
& & \\
& & \\
\end{tabular}

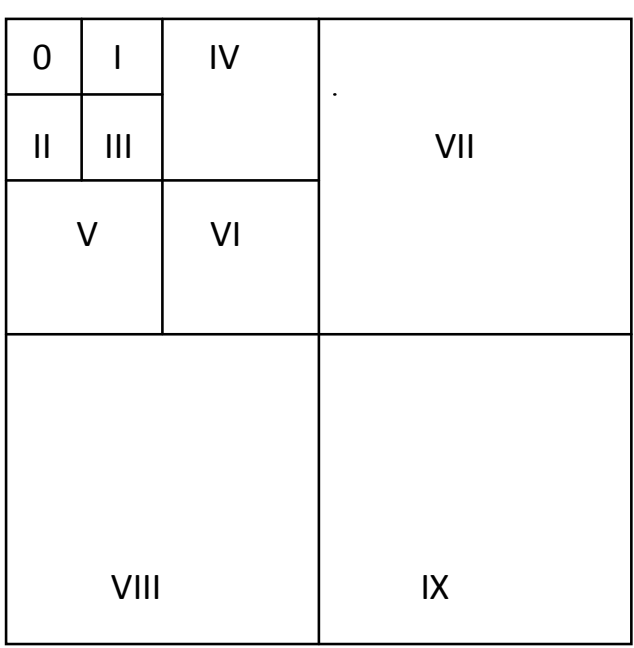

Training Images

Fig 2: Illustrates the learning process

Table 1: Comparison of MSE and PSNR expressed in $\mathrm{db}$

\begin{tabular}{|c|c|c|c|c|}
\hline \multirow{2}{*}{ Wavelet } & \multicolumn{2}{|c|}{ Building } & \multicolumn{2}{c|}{ Face } \\
\cline { 2 - 5 } & MSE & PSNR & MSE & PSNR \\
\hline Haar & 0.0364 & 19.3278 & 0.0341 & 22.0579 \\
\hline Db4 & 0.0043 & 28.5749 & 0.0043 & 31.0984 \\
\hline Coif3 & 0.0061 & 27.0855 & 0.0052 & 30.2629 \\
\hline Bior2.4 & 0.0238 & 21.1761 & 0.0210 & 24.1621 \\
\hline Dmey & 0.0253 & 20.9039 & 0.0227 & 23.8322 \\
\hline
\end{tabular}



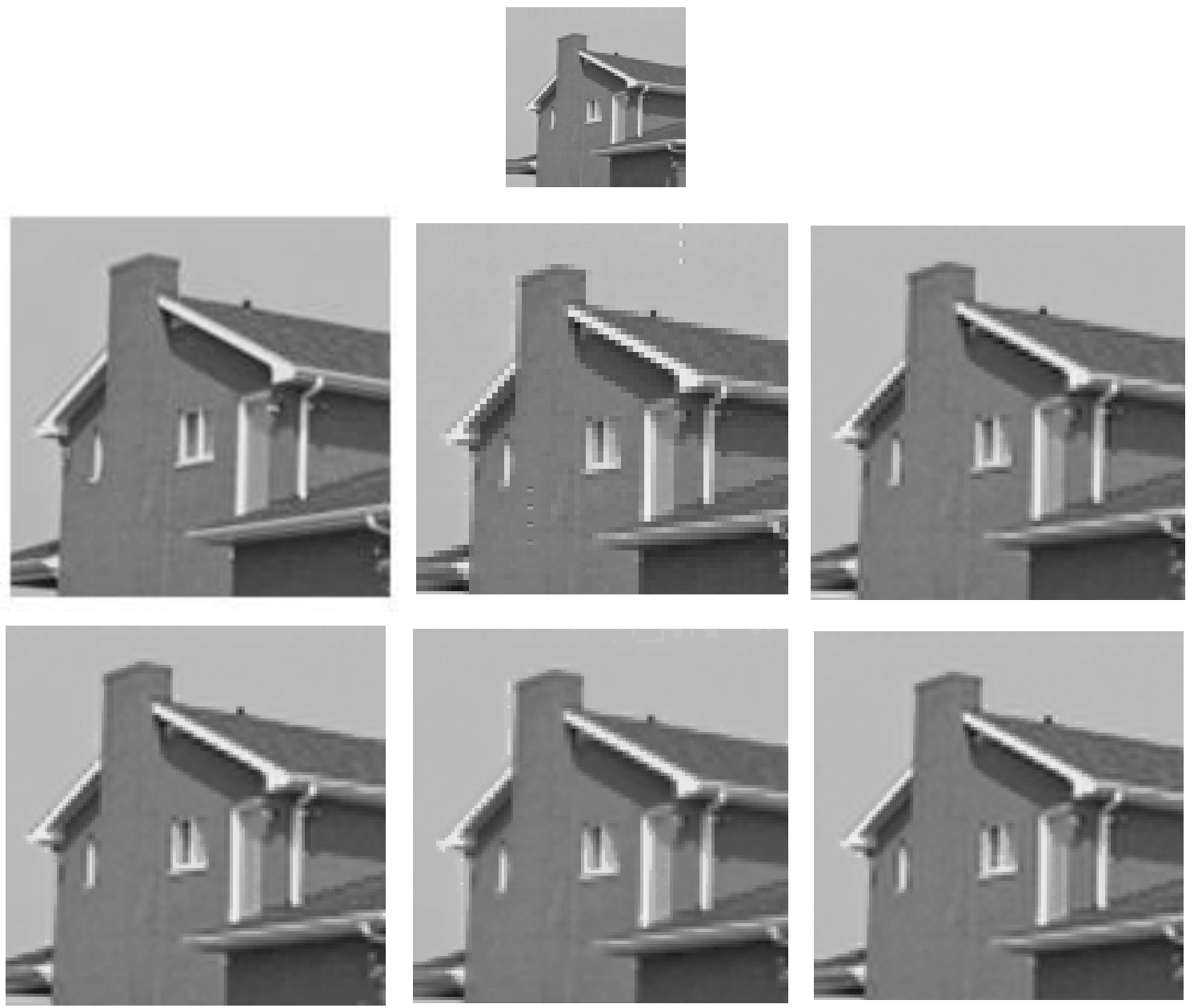

Fig 3: a) Low resolution test Image b) Original High resolution image c) Super resolved using haar d) Super resolved using db4 e) Super resolved using coif3 f) Super resolved using bior2.4 g) Super resolved using dmey
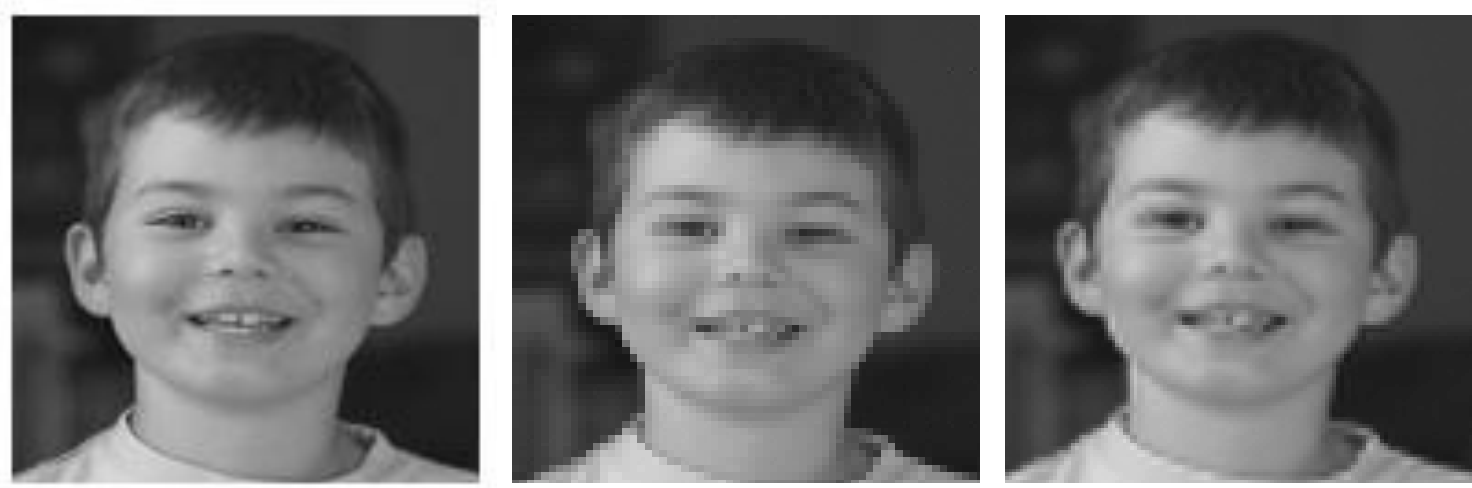

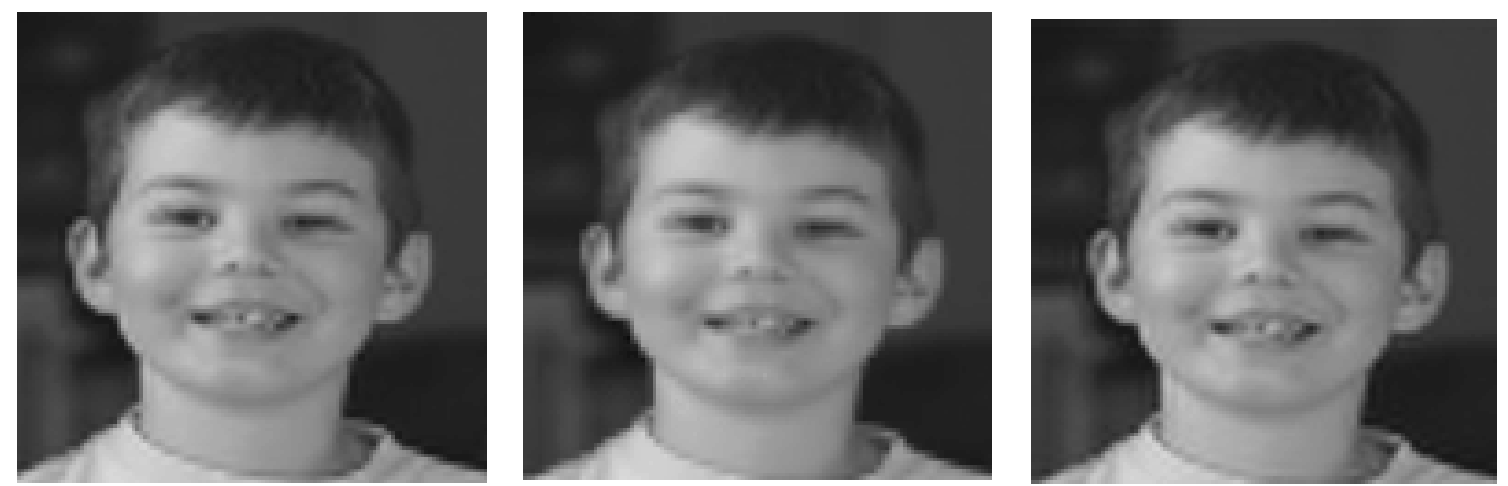

Fig 4: a) Low resolution test Image b) Original High resolution image c) Super resolved using haar d) Super resolved using db4 e) Super resolved using coif3 f) Super resolved using bior 2.4 g) Super resolved using dmey
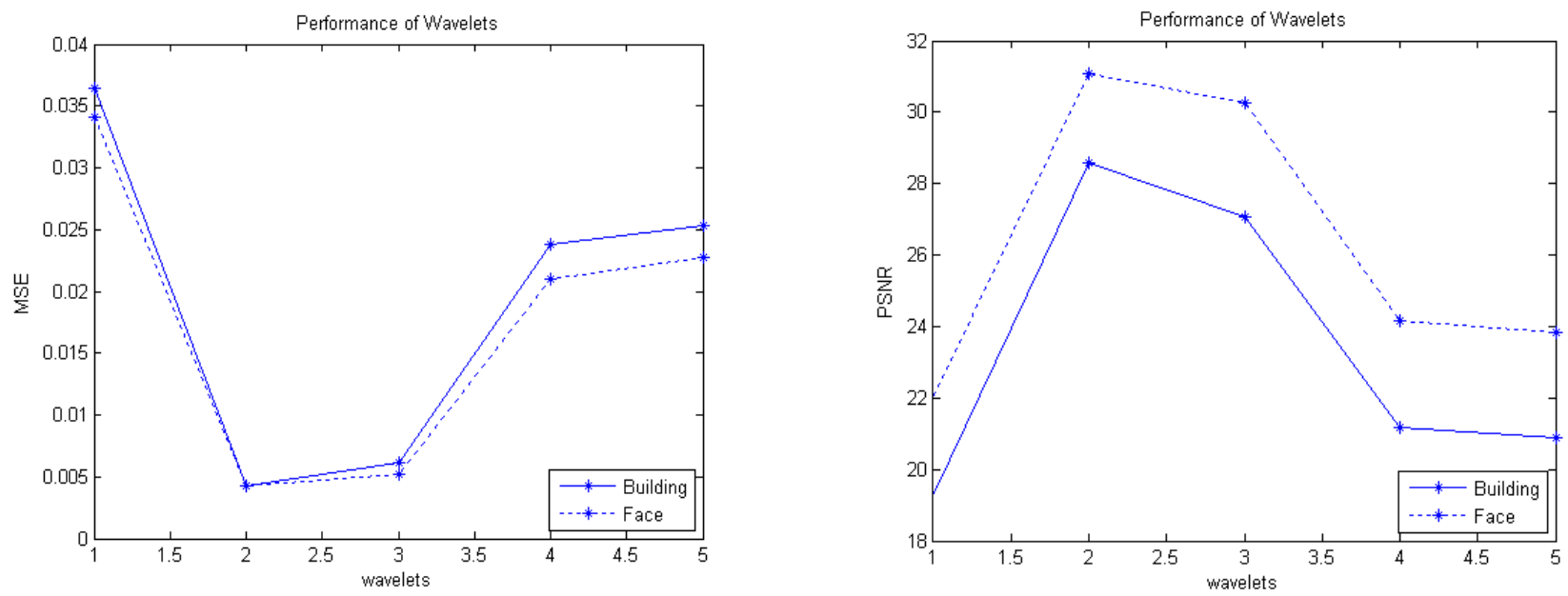

Fig 5: a) MSE vs. Wavelets b) PSNR vs. Wavelets

\section{REFERENCES}

[1] S.Park, M.Park and Kang," Super-Resolution image Reconstruction: A Tech. Overviwe," IEEE Signal Processing Magazine. Vol.03,pp 21-36, May 2003.

[2] M. Irani and S. Peleg," Improving Resolution by Image Registration," CVGIP: Graphical Models and Image Processing, vol.53, pp. 231-239, March 1991..

[3] P. Vandewalle, L. Sbaiz, S. Süsstrunk and M. Vetterli, Registration of Aliased Images for Super-Resolution Imaging, Proc. SPIE/IS\&T Visual Communications and Image Processing Conference, Vol. 6077, pp. 13-23, 2006.

[4] D.Rajan and S.Chaudhuri," Generation of Superresolution Images from Blurred Observations Using an MRF Model," J. Mathematical Imaging and Vision, vol. 16, pp. 5-15, 2002..

[5] D.Capel and A.Zisserman,"Super-resolution form multiple views using learnt image model," in Proc. of IEEE Computer Society Conf. on Computer Vision and Pattern Recognition(CVPR'01),vol.2,pp.II-627634,Kausi,Hawaii,USA,Dec.2001
[6] M.V.Joshi and S.Chaudhuri," A learning based method for image super-resolution from zoomed observations," Proc. of $5^{\text {th }}$ Int. Conf. on Advances in Pattern Recognition (ICAPR'03) pp.179-182, Calcutta, India, Dec.2003.

[7] J. M. Shapiro, "Embedded image coding using zerotrees of wavelet coefficients," IEEE Transactions on Signal Processing,vol. 41, no. 12, pp. 3445-3462, 1993

[8] C.V.Jiji, M.V.Joshi and S.Chaudhuri," Single-frame image super-resolution using learned wavelet coefficients" International Journal of Imaging Systems and Technology, vol.14, no.3, pp.105-112, 2004

[9] Rao R M \& Bopardikar A S, Wavelet Transform: Introduction to Theory and Application (Addison Wesley Longman Inc.) 1998.

[10] I.Daubechies,"Ten Lectures on Wavelets," Philadelphia, PA: Society for Industrial and Applied Mathematics (SIAM),1992,Capital CityPress 